\title{
Gnedenko-Raikov's Theorem Fails for Exchangeable Sequences
}

\author{
George Stoica ${ }^{1 *}$, Deli $\mathrm{Li}^{2}$ \\ ${ }^{1}$ Department of Mathematical Sciences, University of New Brunswick, Saint John, Canada \\ ${ }^{2}$ Department of Mathematical Sciences, Lakehead University, Thunder Bay, Canada \\ Email: "stoica@unb.ca
}

Received July 17, 2012; revised August 17, 2012; accepted August 25, 2012

\begin{abstract}
We study the connection between the central limit theorem and law of large numbers for exchangeable sequences, and provide a counterexample to the Gnedenko-Raikov theorem for such sequences.
\end{abstract}

Keywords: Exchangeable Sequences; Central Limit Theorem; Weak Law of Large Numbers

The celebrated Gnedenko-Raikov theorem states that sums of independent, infinitesimal random variables are asymptotically normal if and only if the sum of squares, centered at truncated means, is relatively stable. The following variant for i.i.d. random variables has been recently proved in [1]:

Theorem 1. Let $\left\{\xi_{n}, n \geq 1\right\}$ be i.i.d. random variables with mean zero, and $\left\{b_{n}, n \geq 1\right\}$ a sequence of positive reals increasing to $+\infty$. Then

$$
\frac{\xi_{1}+\cdots+\xi_{n}}{b_{n}} \rightarrow N(0,1) \text { in distribution }
$$

if and only if

$$
\frac{\xi_{1}^{2}+\cdots+\xi_{n}^{2}}{b_{n}^{2}} \rightarrow 1 \text { in probability. }
$$

A classical extension of independence is exchangeability, and in this context we shall prove that the Gnedenko-Raikov theorem fails. First, let us recall the basic facts. A sequence of random variables $\left\{X_{n}, n \geq 1\right\}$ on the probability space $(\Omega, F, P)$ is said to be exchangeable if for each $n$,

$$
P\left(X_{1} \leq x_{1}, \cdots, X_{n} \leq x_{n}\right)=P\left(X_{\pi(1)} \leq x_{1}, \cdots, X_{\pi(n)} \leq x_{n}\right)
$$

for any permutation $\pi$ of $\{1,2, \cdots, n\}$ and any $x_{i} \in \mathbb{R}, i=1, \cdots, n$. Two trivial examples are i.i.d. random variables and totally determined random variables $\{X, X, \cdots\}$. Two nontrivial but simple examples are $\left\{X+r_{n}, n \geq 1\right\}$ and $\left\{Y \cdot r_{n}, n \geq 1\right\}$ where the $r_{n}$ 's are i.i.d. and independent of $X$ or $Y$, respectively.

*Corresponding author.
By de Finetti's theorem, an infinite sequence of exchangeable random variables is conditionally i.i.d. given either the tail $\sigma$-field of $\left\{X_{n}, n \geq 1\right\}$ or the $\sigma$-field $G$ of permutable events. Furthermore, there exists a regular conditional distribution $P^{\omega}$ for $\left\{X_{n}, n \geq 1\right\}$ given $G$ such that for each $\omega \in \Omega$ the coordinate random variables $\left\{\xi_{n} \equiv \xi_{n}^{\omega}\right\}$, called mixands, of the probability space $\left(\mathbb{R}^{\infty}, B^{\infty}, P^{\omega}\right)$ are i.i.d. Namely, for each natural number $n$, any Borel function $f: \mathbb{R}^{n} \rightarrow \mathbb{R}$, and any Borel set $B$ on $\mathbb{R}$,

$$
P\left(f\left(X_{1}, \cdots, X_{n}\right) \in B\right)=\int_{\Omega} P^{\omega}\left(f\left(\xi_{1}, \cdots, \xi_{n}\right) \in B\right) \mathrm{d} P .
$$

The following central limit theorem for exchangeable sequences has been proved in [2]:

Theorem 2. Let $\left\{X_{n}, n \geq 1\right\}$ be a sequence of exchangeable random variables. Then there exist constants $a_{n}, b_{n}$ with $b_{n} \rightarrow \infty$, such that

$\frac{X_{1}+\cdots+X_{n}-a_{n}}{b_{n}} \rightarrow N(0,1) \quad$ in distribution if and only if there exists a positive sequence $\varepsilon_{n} \searrow 0$ such that

$$
n P^{\omega}\left(\left|\xi_{1}\right|>\varepsilon_{n} b_{n}\right) \rightarrow 0 \text { in probability. }
$$

and either $b_{n} / \sqrt{n}$ is slowly varying with

$$
\frac{v_{n}(\omega)}{b_{n}} \rightarrow 1, \frac{a_{n}(\omega)-a_{n}}{b_{n}} \rightarrow 0 \text { in probability }
$$

or $b_{n} / n$ is slowly varying with

$$
\frac{v_{n}(\omega)}{b_{n}} \rightarrow 0 \text { in probability, }
$$




$$
\frac{a_{n}(\omega)-a_{n}}{b_{n}} \rightarrow N(0,1) \text { in distribution, }
$$

where

$$
a_{n}(\omega):=n E^{\omega}\left(\xi_{1} 1_{\left(\left|\xi_{1}\right| \leq \varepsilon_{n} b_{n}\right)}\right)
$$

and

$$
v_{n}^{2}(\omega)=n\left[E^{\omega}\left(\xi_{1}^{2} 1_{\left(|\xi| \xi \mid \leq \varepsilon_{n} b_{n}\right)}\right)-\left(E^{\omega}\left(\xi_{1} 1_{\left(|\xi \xi| \mid \varepsilon_{n} b_{n}\right)}\right)\right)^{2}\right] .
$$

In the above theorem, the case where $b_{n} / \sqrt{n}$ is slowly varying characterizes the situation when the classical central limit theorem holds for the mixands, whereas the case where $b_{n} / n$ is slowly varying characterizes the situation when the law of large numbers holds for the mixands and those limits have a standard normal distribution. Recently, we "cleaned" the latest statement and proved in [3] the following variant of the law of large numbers for exchangeable sequences:

Theorem 3. Let $\left\{X_{n}, n \geq 1\right\}$ be a sequence of exchangeable random variables and $\left\{b_{n}, n \geq 1\right\}$ a sequence of positive reals increasing to $+\infty$, that satisfy the following conditions:

$$
n P^{\omega}\left(\left|\xi_{1}\right|>b_{n}\right) \rightarrow 0 \text { in probability }
$$

and

$$
\tilde{v}_{n}(\omega) b_{n} \rightarrow 0 \text { in probability, }
$$

where

$$
\tilde{v}_{n}^{2}(\omega)=n\left[E^{\omega}\left(\xi_{1}^{2} 1_{\left(\left|\xi_{1}\right| \leq b_{n}\right)}\right)-\left(E^{\omega}\left(\xi_{1} 1_{\left(\left|\xi_{1}\right| \leq b_{n}\right)}\right)\right)^{2}\right] .
$$

Then

$$
\frac{X_{1}+\cdots+X_{n}-\tilde{a}_{n}(\omega)}{b_{n}} \rightarrow 0 \text { in probability, }
$$

where

$$
\tilde{a}_{n}(\omega)=n E^{\omega}\left(\xi_{1} 1_{\left(|\xi| \leq b_{n}\right)}\right) .
$$

Unless the sequence $\left\{X_{n}, n \geq 1\right\}$ is i.i.d., the converse in the above theorem is not true; more is needed, see [4].

We are now ready to provide the counterexample mentioned in the introduction. It will rely on both Theorems 2 and 3 , and some specific constants $\left\{b_{n}, n \geq 1\right\}$. More precisely, we have:

Theorem 4. Let $\left\{X_{n}, n \geq 1\right\}$ be a sequence of exchangeable random variables and $\left\{b_{n}, n \geq 1\right\}$ a sequence of norming constants that satisfy the following condition:

$$
n P^{\omega}\left(\left|\xi_{1}\right|>\varepsilon_{n} b_{n}\right) \rightarrow 0 \text { in probability, }
$$

where $\left\{\varepsilon_{n}, n \geq 1\right\}$ is the sequence appearing in Theorem 2.

1) Assume that the sequence $\left\{\varepsilon_{n} b_{n}, n \geq n_{0}\right\}$ is nondecreasing for some $n_{0} \geq 1$ and satisfies

$$
\sum_{k=1}^{n} \frac{\varepsilon_{k}^{2} b_{k}^{2}}{k^{2}} \leq c \frac{\varepsilon_{n}^{2} b_{n}^{2}}{n}
$$

for all $n \geq 1$ and some constant $c>0$. Then

$$
\frac{\left(X_{1}^{2}+\cdots+X_{n}^{2}\right)}{b_{n}^{2}} \rightarrow 0 \text { in probability. }
$$

2) If $b_{n} / n$ and $\varepsilon_{n} / n^{\rho-1}$ are slowly varying for some $1 / 2<\rho<1$, then

$$
X_{1}+\cdots+X_{n} b_{n} \rightarrow N(0,1) \text { in distribution, }
$$

and the Gnedenko-Raikov theorem fails in this case.

Proof of Theorem 4. 1) Under the assumptions on the sequence $\left\{\varepsilon_{n} b_{n}, n \geq n_{0}\right\}$ and according to [5], p. 680, we have that

$$
n E^{\omega}\left(\xi_{1}^{2} 1_{\left(\left|\xi_{1}\right| \leq \varepsilon_{n} b_{n}\right)}\right) \varepsilon_{n}^{2} b_{n}^{2} \rightarrow 0 \text { in probability. }
$$

Also, cf. Section 2 in [5], we have that $\varepsilon_{n} b_{n} \rightarrow+\infty$ and $b_{n} \rightarrow+\infty$. These facts imply that

$$
\frac{n E^{\omega}\left(\xi_{1}^{2} 1_{\left(\left|\xi_{1}\right| \leq \varepsilon_{n} b_{n}\right)}\right)}{b_{n}^{2}} \rightarrow 0 \text { in probability. }
$$

Taking into account the following identity (with the notations in Theorem 2):

$$
v_{n}^{2}(\omega)=n\left[E^{\omega}\left(\xi_{1}^{2} 1_{\left(|\xi| \xi \varepsilon_{n} b_{n}\right)}\right)-a_{n}^{2}(\omega) / n^{2}\right],
$$

which gives

$$
\frac{n E^{\omega}\left(\xi_{1}^{2} 1_{\left(\left|\xi_{1}\right| \leq \varepsilon_{n} b_{n}\right)}\right)}{b_{n}^{2}}=\frac{a_{n}^{2}(\omega)}{n b_{n}^{2}}+\frac{v_{n}^{2}(\omega)}{b_{n}^{2}}
$$

from formula (3) it follows that

$$
\begin{aligned}
& a_{n}^{2}(\omega) / n b_{n}^{2} \rightarrow 0 \text { in probability, } \\
& \text { and } v_{n} / b_{n} \rightarrow 0 \text { in probability. }
\end{aligned}
$$

Now let $\varepsilon>0$ be given. By formula (1) and the triangle inequality we have

$$
\begin{aligned}
& P\left(\left|\left(X_{1}^{2}+\cdots+X_{n}^{2}\right) / b_{n}^{2}\right|>\varepsilon\right) \\
= & \int_{\Omega} P^{\omega}\left(\left|\left(\xi_{1}^{2}+\cdots+\xi_{n}^{2}\right) / b_{n}^{2}\right|>\varepsilon\right) \mathrm{d} P \\
\leq & \int_{\Omega} P^{\omega}\left(\left|\frac{1}{b_{n}^{2}} \sum_{k=1}^{n} \xi_{k}^{2} 1_{\left(\left|\xi_{k}\right| \leq \varepsilon_{n} b_{n}\right)}\right|>\frac{\varepsilon}{2}\right) \mathrm{d} P \\
& +\int_{\Omega} P^{\omega}\left(\left|\frac{1}{b_{n}^{2}} \sum_{k=1}^{n} \xi_{k}^{2} 1_{\left(\left|\xi_{k}\right| \varepsilon_{n} b_{n}\right)}\right|>\frac{\varepsilon}{2}\right) \mathrm{d} P .
\end{aligned}
$$


Using (2), we estimate the first term in the right hand side of (5) as follows:

$$
\begin{aligned}
& P^{\omega}\left(\left|\frac{1}{b_{n}^{2}} \sum_{k=1}^{n} \xi_{k}^{2} 1_{\left(\left|\xi_{k}\right| \leq \varepsilon_{n} b_{n}\right)}\right|>\frac{\varepsilon}{2}\right) \\
& \leq P^{\omega}\left(\bigcup_{k=1}^{n}\left(\left|\xi_{k}\right|>\varepsilon_{n} b_{n}\right)\right) \\
& \leq n P^{\omega}\left(\left|\xi_{k}\right|>\varepsilon_{n} b_{n}\right) \rightarrow 0 \text { in probability. }
\end{aligned}
$$

We then break down the second term in the right hand side of (5) as follows:

$$
\begin{aligned}
& P^{\omega}\left(\left|\frac{1}{b_{n}^{2}} \sum_{k=1}^{n} \xi_{k}^{2} 1_{\left(\left|\xi_{k}\right| \leq \varepsilon_{n} b_{n}\right)}\right|>\frac{\varepsilon}{2}\right) \\
& \leq P^{\omega}\left(\left|\frac{1}{b_{n}^{2}} \sum_{k=1}^{n} \xi_{k}^{2} 1_{\left(\left|\xi_{k}\right| \varepsilon_{n} b_{n}\right)}-\frac{1}{b_{n}^{2}} \sum_{k=1}^{n} E^{\omega}\left(\xi_{k}^{2} 1_{\left(\left|\xi_{k}\right| \leq \varepsilon_{n} b_{n}\right)}\right)\right|>\frac{\varepsilon}{4}\right) \\
& +P^{\omega}\left(\left|\frac{1}{b_{n}^{2}} \sum_{k=1}^{n} E^{\omega}\left(\xi_{k}^{2} 1_{\left(\left|\xi_{k}\right| \leq \varepsilon_{n} b_{n}\right)}\right)-\frac{n}{b_{n}^{2}}\left(E^{\omega} \xi_{k} 1_{\left(\left|\xi_{k}\right| \leq \varepsilon_{n} b_{n}\right)}\right)^{2}\right|>\frac{\varepsilon}{8}\right) \\
& +P^{\omega}\left(\left|\frac{n}{b_{n}^{2}}\left(E^{\omega} \xi_{k} 1_{\left(\left|\xi_{k}\right| \leq \varepsilon_{n} b_{n}\right)}\right)^{2}\right|>\frac{\varepsilon}{8}\right) .
\end{aligned}
$$

Using (4), we have

$$
\begin{aligned}
& P^{\omega}\left(\left|\frac{1}{b_{n}^{2}} \sum_{k=1}^{n} \xi_{k}^{2} 1_{\left(\left|\xi_{k}\right| \leq \varepsilon_{n} b_{n}\right)}-\frac{1}{b_{n}^{2}} \sum_{k=1}^{n} E^{\omega}\left(\xi_{k}^{2} 1_{\left(\left|\xi_{k}\right| \leq \varepsilon_{n} b_{n}\right)}\right)\right|>\frac{\varepsilon}{4}\right) \\
& \leq \frac{16}{\varepsilon^{2}} \frac{1}{b_{n}^{4}} n \operatorname{Var} \xi_{1}^{2} 1_{\left(\left|\xi_{\mid}\right| \leq \varepsilon_{n} b_{n}\right)} \leq \frac{16}{\varepsilon^{2}} \frac{\varepsilon_{n}^{2} v_{n}^{2}(\omega)}{b_{n}^{2}} \\
& \rightarrow 0 \text { in probability. }
\end{aligned}
$$

Also, cf. (4),

$$
\begin{aligned}
& P^{\omega}\left(\left|\frac{1}{b_{n}^{2}} \sum_{k=1}^{n} E^{\omega}\left(\xi_{k}^{2} 1_{\left|\xi_{k}\right|} \leq \varepsilon_{n} b_{n}\right)-\frac{n}{b_{n}^{2}}\left(E^{\omega} \xi_{k} 1_{\left(\left|\xi_{k}\right| \leq \varepsilon_{n} b_{n}\right)}\right)^{2}\right|>\frac{\varepsilon}{8}\right) \\
& =1_{\left(v_{n}^{2}(\omega) / b_{n}^{2}>\varepsilon / 8\right)} \rightarrow 0 \text { in probability, }
\end{aligned}
$$

and, again cf. (4),

$$
\begin{aligned}
& P^{\omega}\left(\left|\frac{n}{b_{n}^{2}}\left(E^{\omega} \xi_{k} 1_{\left(\left|\xi_{k}\right| \leq \varepsilon_{n} b_{n}\right)}\right)^{2}\right|>\frac{\varepsilon}{8}\right) \\
& =1_{\left(\left|a_{n}^{2}(\omega) / n b_{n}^{2}\right|>\varepsilon / 8\right)} \rightarrow 0 \text { in probability. }
\end{aligned}
$$

From (5)-(10) we deduce that $\left(X_{1}^{2}+\cdots+X_{n}^{2}\right) / b_{n}^{2} \rightarrow 0$ in probability.
Now, let us prove 2). If $b_{n} / n$ is slowly varying, and using (4), Theorems 2 and 3 imply that $\left(X_{1}+\cdots+X_{n}\right) / b_{n} \rightarrow N(0,1)$ in distribution. If, in addition, $\varepsilon_{n} / n^{\rho-1}$ is slowly varying for some $1 / 2<\rho<1$, then the hypotheses on the sequence $\left\{\varepsilon_{n} b_{n}, n \geq n_{0}\right\}$ in part 1) of Theorem 4 are satisfied cf. section 2 in [5], hence the Gnedenko-Raikov theorem fails in this case.

Remark. It is worth noting that the Gnedenko-Raikov theorem is valid in the case where $b_{n} / \sqrt{n}$ is slowly varying in Theorem 2, as well as in both self-normalized central limit theorem [6] and self-normalized law of large numbers [7] for exchangeable sequences. This is why the counterexample in Theorem 4 above was rather hard to get.

The research of George Stoica and Deli Li was partially supported by grants from the Natural Sciences and Engineering Research Council of Canada.

\section{REFERENCES}

[1] A. Gut, "Gnedenko-Raikov's Theorem, Central Limit Theory, and the Weak Law of Large Numbers," Statistics and Probability Letters, Vol. 76, No. 17, 2006, pp. 19351939. doi:10.1016/j.spl.2006.04.042

[2] M. Klass and H. Teicher, "The Central Limit Theorem for Exchangeable Random Variables without Moments," Annals of Probability, Vol. 15, No. 1, 1987, pp. 138-153. doi:10.1214/aop/1176992260

[3] G. Stoica and D. Li, "On the Kolmogorov-Feller Law for Exchangeable Random Variables," Statistics and Probability Letters, Vol. 80, No. 9-10, 2012, pp. 899-902. doi:10.1016/j.spl.2010.01.025

[4] G. Stoica, "An Extension of the Weak Law of Large Numbers for Exchangeable Sequences," Acta Applicandae Mathematicae, Vol. 109, No. 3, 2010, pp. 759-763. doi:10.1007/s10440-008-9344-x

[5] M. V. Kruglov, "A Generalization of the Weak Law of Large Numbers," Stochastic Analysis and Applications, Vol. 29, No. 4, 2011, pp. 674-683. doi:10.1080/07362994.2011.581099

[6] X. Jiang and M. G. Hahn, "Empirical Central Limit Theorems for Exchangeable Random Variables," Statistics and Probability Letters, Vol. 59, No. 1, 2002, pp. 75-81. doi:10.1016/S0167-7152(02)00204-3

[7] G. Stoica, "A Self-Normalized Weak Law of Large Numbers for Exchangeable Sequences," Advances and Applications in Statistics, Vol. 25, No. 2, 2011, pp. 103-108. 